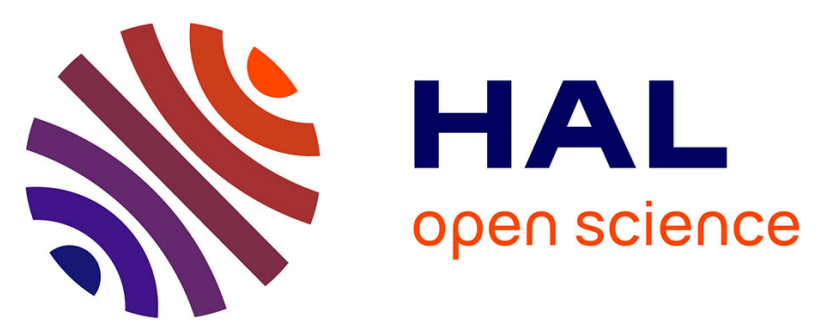

\title{
Synthesis on High-Fidelity Numerical simulation of a morphing A320 wing in subsonic speeds and sensitivity evaluation
}

Nikolaos Simiriotis, Marianna Braza, Konstantinos Diakakis, Georgios

Tzabiras, Gurvan Jodin, Jean-François Rouchon, Abderahmane Marouf, Yannick Hoarau, Felix Kramer

\section{To cite this version:}

Nikolaos Simiriotis, Marianna Braza, Konstantinos Diakakis, Georgios Tzabiras, Gurvan Jodin, et al.. Synthesis on High-Fidelity Numerical simulation of a morphing A320 wing in subsonic speeds and sensitivity evaluation. AIAA Aviation 2019 Forum, 2019, Dallas, United States. 10.2514/6.20192911. hal-03106600

\author{
HAL Id: hal-03106600 \\ https://hal.science/hal-03106600
}

Submitted on 11 Jan 2021

HAL is a multi-disciplinary open access archive for the deposit and dissemination of scientific research documents, whether they are published or not. The documents may come from teaching and research institutions in France or abroad, or from public or private research centers.
L'archive ouverte pluridisciplinaire $\mathbf{H A L}$, est destinée au dépôt et à la diffusion de documents scientifiques de niveau recherche, publiés ou non, émanant des établissements d'enseignement et de recherche français ou étrangers, des laboratoires publics ou privés. 


\title{
Synthesis on High-Fidelity Numerical simulation of a morphing A320 wing in subsonic speeds and sensitivity evaluation
}

\author{
N. Simiriotis* and M. Braza ${ }^{\dagger}$ \\ IMFT UMR 5502, 2 Allée du Professeur Camille Soula, 31400, Toulouse, France.
}

K. Diakakis ${ }^{\ddagger}$ and G. Tzabiras ${ }^{\S}$

National Technical University of Athens (NTUA), 9 Heroon Polytechneiou str., GR15780, Athens, Greece.

G. Jodin ${ }^{\mathrm{II}}$ and J.F. Rouchon"

LAPLACE UMR 5213, 2 rue Charles Camichel, 31071, Toulouse, France.

\author{
A. Marouf** and Y. Hoarau ${ }^{\dagger \dagger}$ \\ ICUBE UMR7357, 300 Bd Sébastien Brant, 67400, Strasbourg, France.
}

\begin{abstract}
F. Kramert
CFD Software Entwicklungs - und Forschungsgesellschaft mbH, Wolzogenstraße 4, 14163, Berlin, Germany.
\end{abstract}

This contribution examines the effect of trailing-edge region deformation and vibration on the turbulent structures developing around and in the wake of a supercritical wing of A320 type. A multi-parametric analysis covers the effects that amplitude and frequency ranges have on the aerodynamic performance. This study shows the capacity of morphing to increase lift, decrease drag and drastically change the instability modes developed in the flow and are associated to aerodynamic noise. The work presented has been carried out numerically and is accompanied by experimental results from tests carried out in the S4 wind tunnel of IMFT by means of Time-Resolved Particle Image Velocimetry (TRPIV). The Reynolds number is 1 Million. The study is carried out in the low subsonic regime at an incidence angle of $10^{\circ}$, corresponding to take-off/landing flight phases. The effects on the aerodynamic coefficients thanks to manipulation of specific vortex dynamics in the shear layers and the wake are discussed and optimal frequency/amplitude ranges for the vibrations are quantified.

\section{Nomenclature}

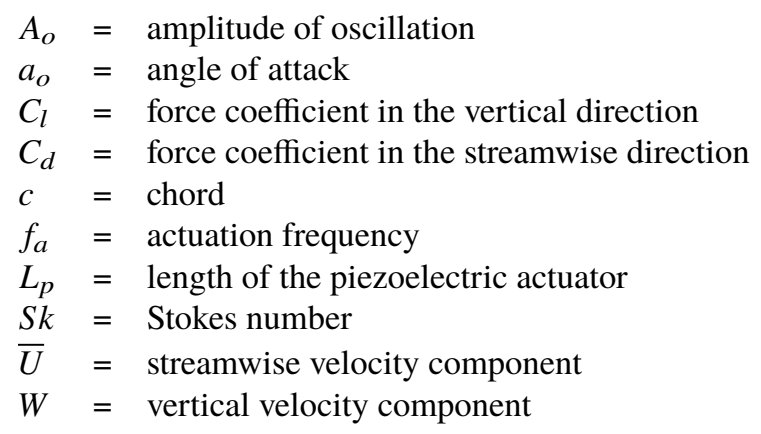

*PhD Candidate, DGA/CNRS, nikolaos.simiriotis@imft.fr.

† Research Director, CNRS/IMFT, marianna.braza@imft.fr.

$¥$ PhD Candidate, Laboratory of Aerodynamics, diak@ fluid.mech.ntua.gr.

§Professor, School of Naval Architecture and Marine Engineering, tzab@ fluid.mech.ntua.gr.

IDoctor, LAPLACE/IMFT, gurvan.jodin@laposte.net.

"Professor, ENSEEIHT, rouchon@laplace.univ-tlse.fr.

**PhD candidate, ICUBE/IMFT, abderahmane.marouf@etu.unistra.fr.

${ }^{\dagger}$ Professor, Faculty of Physics and Engineering, University of Strasbourg, hoarau@ unistra.fr.

\#octor, CFDB, felix.kramer@cfd-berlin.com. 
$x_{o}=$ starting position of the piezoelectric actuator

$y^{+}=$the non-dimensional wall distance

\section{Introduction}

$T^{H E}$ present article aims at highlighting the capacity of morphing to increase lift, decrease drag and reduce the amplitude of instability modes associated to aerodynamic noise. Morphing refers to the adaptation of the real-time shape and vibrational behavior of the aerodynamic surface. In this context, a multi-disciplinary research platform involving the IMFT (Institut de Mécanique des Fluides de Toulouse) and LAPLACE (Laboratoire Plasma et Conversion d'Energie) and laboratories has been continuously working in this topic. By this synergistic platform among six Institutes in Toulouse (www.smartwing.org), coordinated by IMFT and in close collaboration with AIRBUS Emerging Technologies and Concepts Toulouse, advanced electroactive morphing designs for the wings of the future are currently being developed and studied in the context of the European research program of H2020, "Smart Morphing \& Sensing for Aeronautical configurations" (www.smartwing.org/SMS/EU). In particular, the hybrid morphing concept, partly bio-inspired, was developed ([1], [2]) to operate at various time scales by utilizing different classes of electroactive actuators. When realized simultaneously, modifications of turbulence can be achieved at multiple scales.

This study focuses on the electroactive morphing effects at high Reynolds numbers (order of $10^{6}$ ) by means of numerical simulation. Previous studies in the literature were carried out at low Reynolds numbers [3], [4] examined part of the flow characteristics and are quite limited and not operating in multiple scales. Past papers have also examined the forcing of the trailing of a wing [5] also in lower Reynolds number. The flow in the supercritical Reynolds range presents complex vortex dynamics and interactions with the solid structure, calling for specific attention in order to produce successful morphing effects. High Reynolds dynamics change the general flow behavior and the morphing practices and targets. In the present context, the electro-active morphing is a more general strategy than standard flow control techniques as it creates interactions among structural dynamics and turbulence manipulation. A previous experimental study in [2] has presented a first analysis of the morphing effects.
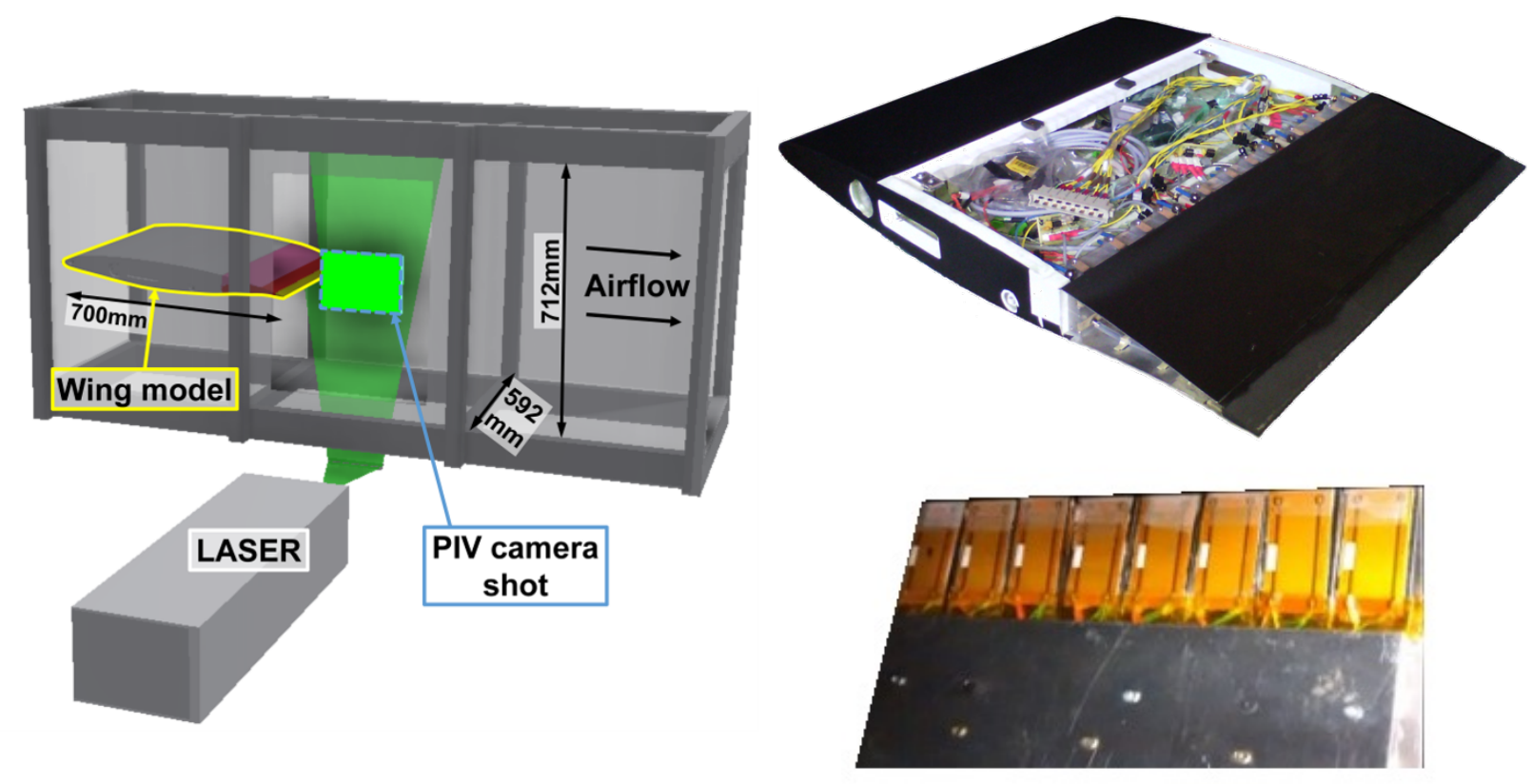

Fig. 1 Schematic representation of the experimental test section of the S4 wind tunnel of IMFT (left) with the Airbus-A320 wing (right-top) mounted on [2]; the prototype bears the HFVTE actuators (right-bottom) on the trailing edge along the span.

The considered prototype is a design of an electroactive hybrid morphing wing. The baseline airfoil is the wing section of Airbus-A320, with a chord of $700 \mathrm{~mm}$ and a span of $592 \mathrm{~mm}$ placed at an incident angle of $10^{\circ}$ in the subsonic wind tunnel S4 of IMFT (see Fig. 1). It is equipped with both a camber control system and a High Frequency Vibrating 
Trailing Edge (HFVTE) actuators. The working principle of the camber control relies on distributed structure embedded actuators. Shape Memory Alloy (SMA) wires are placed under the upper and lower sides of the aluminum sheets constituting the skin of the wing. The selected SMA wires were made of Nickel-Titanium alloys and their properties are controlled by a change in temperature [6]. The SMA wires are capable of providing high deformations of order $25-30 \%$ of the chord at low frequencies (order of $1 \mathrm{~Hz}$ ). The camber control actuators were sized and implemented on a length covering the last $30 \%$ of the chord, corresponding to the actual flap placement.

The HFVTE actuators are composed of metallic substrates positioned in between Macro Fiber Composite (MFC) piezoelectric patches. The MFC patches are LZT piezoelectric fibers and electrode networks encapsulated within epoxy. When supplied by a voltage, the patches stretch out and generate bending. The Multi-Fiber composite piezoelectric actuators were glued on both sides of a metallic substrate and placed in the trailing edge region. The whole ensemble is covered by a flexible molded silicon in order to retain the trailing edge shape and limit the impact on the actuator's performance. The active length of the HFVTE is $35 \mathrm{~mm}$ chord-wise. This implementation allows for quasi-static tip deformation amplitude of $0.5 \mathrm{~mm}-1 \mathrm{~mm}$ even in higher frequencies (up to $400 \mathrm{~Hz}$ ). For further details, related to the design and the electromechanical characterization of the electroactive morphing wing the reader is invited to refer to [7].

In this analysis, only the electroactive morphing effects of the HFVTE actuation will be studied. This will highlight the effects coming from the vibrating trailing edge in the context of the hybrid morphing. In addition, a shape optimization is included in order to evaluate the sensitivity of the lift variation due to any surface deformation. Time Resolved Particle Image Velocimetry (TRPIV) measurements will also be included in this paper for the validation of the numerical approach. For a detailed description of the experimental equipment and procedure the reader should refer to [2]. The measurement of the unsteady velocity fields by means of Time-Resolved PIV (TRPIV) was realized with the contribution of the Signal and Image processing service of IMFT (S. Cazin and M. Marchal). The post-processing of the raw PIV results was carried out with the CPIV-IMFT open-source software, developed and validated with the contribution of the software services of IMFT (P. Elyakime) for parallel (MPI) post-treatment of the results in supercomputing architectures. It is capable of computing efficiently in terms of computational time, a set of more than 50000 images which has been proven sufficient to obtain statistical convergence of the results.

\section{Numerical Approach}

The time dependent Navier-Stokes equations have been solved under the conservative form in a finite-volume structured grid formulation, using the Navier-Stokes Multi-Block (NSMB) code [8], in both two and three dimensions. The computational domain is subdivided into a number of quadrilateral (2D) and hexahedral (3D) grid cells resulting in a structured mesh. The multi-block strategy is followed in accordance to the parallelization procedure of the code. A separate discretization of the equations in space and time is applied.

Finite volume cells of constant size in time are considered for the discretization in space. A fourth order standard central skew-symmetric spatial scheme with artificial second and fourth order dissipation terms was used for the convection terms and a second-order central scheme for the diffusion terms. For the temporal discretization, dual time-stepping with a second order implicit backward difference scheme is performed [9]. The artificial compressibility method was implemented in the preconditioning of the flow to increase accuracy in the low subsonic regime.

Computational meshes were constructed representing the test section, the dimension of which can be found in figure 1. Two meshes M1 and M2 have been used for the 2D computations. The grid sizes were selected after thorough numerical studies. The M1 includes 300000 finite volume cells. The refined version M2 contains 500000 finite volume cells. The points were added in the streamwise direction both on the wing and in the wake region. For the 3D computations the M1 grid was extruded in the span-wise direction leading to a total mesh size slightly higher than 10 million (M3). The $y^{+}$values, corresponding to the first grid spacing above the wall, were below 1 for all the grids considered. A comparison of the results in respect of these three grids will be presented in section IV

The physical time-step was kept constant in all the computations at $10^{-5}$ giving a $C F L$ (Courant, Friedrichs and Lewy) number around 50. About $60-80$ inner iterations were carried out for each time-step. The increased number of iterations is justified from the low Mach aerodynamics simulated in the cases following. The upper and lower walls of the tunnel were considered by means of both non-slip (zero velocity at the wall) and slip (velocity tangent to the surface) boundary conditions. After numerical tests that indicated no considerable effect due to the respective boundary layers on the wake development, they have been given a slip condition. A velocity inlet and a pressure outlet were used in the respective boundaries of the domain. The boundary conditions, the preconditioning methodology and every computational parameter has been chosen after various numerical tests. For the morphing test cases, where the body dynamically deforms, the unsteady Arbitrary Langrangian Eulerian (ALE) methodology [10] was used for the moving 


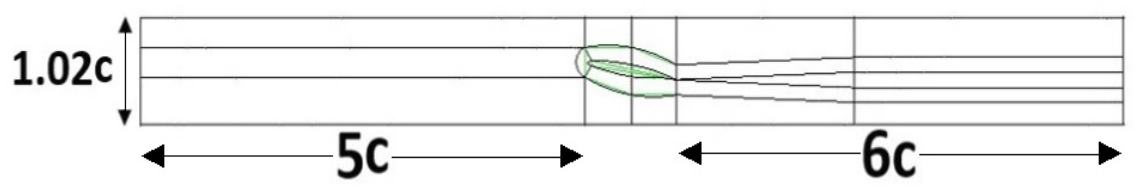

(a) Multi block description of the computational domain.

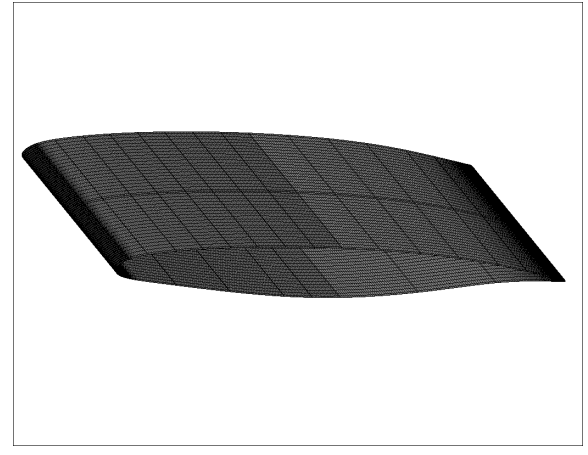

(b) 3D surface meshing of the A320 wing.

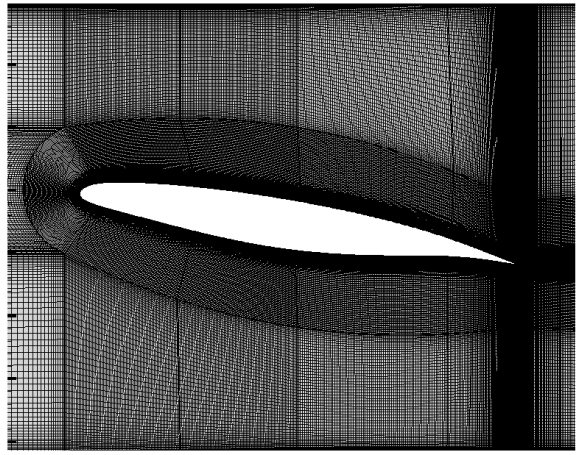

(c) 2D mesh around the A320 wing.

Fig. 2 Meshing of the computational domain.

grid by taking into account the convection of the grid nodes during the deformation. A second-order polynomial was used for the calculation of the imposed deformation of the wing's surface.

For the analysis, the Organized Eddy Simulation (OES) approach [11-13] has been employed. Based on the ensemble (phase) averaging of the flow, this approach is sensitized to allow coherent structures and their related instabilities to develop in the high Reynolds number range and is well adapted for detached flows, both typical of all aeronautical applications. The OES approach is non-inherently 3D and therefore can be applied in 2D simulations with sufficient accuracy in capturing the main coherent structure dynamics and their modification. Therefore, it provides a robust method for capturing physical phenomena and treating near wall turbulence. A more detailed description of the OES can be found in [11] and [12].

In the present work, a shape optimization of the flap and trailing edge deformation was performed using an adjoint flow solver [14] that generates a sensitivity map of the profile. The sensitivity is calculated with respect to the lift as objective function which is first order suitable to the needs of a take-off and/or landing scenario. The multi-purpose in-house flow solver CFDFlux employs a second order Finite Volume discretization on a co-located, block-structured grid. It is highly scalable and specifically improved for acoustic simulations with a pressure-based code. Its discrete adjoint formulation is derived using the TAPENADE [15] software and manually enhanced to increase the code's performance. The adjoint solver uses check-pointing to reduce the extreme storage needs of an unsteady adjoint flow solver.

For the optimization simulations, the compressible unsteady RANS equations are solved with the k-Omega SST turbulence model. The computational 2D C-type grid M4 has 195.000 cells. To ensure reasonable results, multiple constraints were enforced on the free node parametrization: The transition from the deformed part to the fixed part of the profile is realized by blending the step size of the shape deformation during the optimization towards zero. In the same manner, the trailing edge is forced on a circle to keep the chord length fixed. Additionally, some constraints are implemented to preserve the smoothness of the surface.

\section{Validation of the Numerical Approach}

In this section both numerical and experimental results of the non-morphed wing (static case) are examined. For all the results following the wing is mounted with an incidence angle of $10^{\circ}$. The chord of the prototype was constant and equal to $0.7 \mathrm{~m}$. The incoming velocity was held constant $(21.5 \mathrm{~m} / \mathrm{s})$ which for the reference values of temperature $(293 \mathrm{~K})$ and pressure $(101325 \mathrm{~Pa})$ gives a Reynolds number of 1 million. The turbulence intensity of the inlet section of the wind tunnel was estimated at about $0.1 \%$ of the free stream velocity. For the numerical tests, a greater value (close to $1 \%$ ) was used to establish matching levels of turbulence around the wing. 
For the experiments, the velocity variation was evaluated to be below $1.5 \%$ while the blockage ratio was found to be acceptable as long as the focus is the relative effects of the morphing application effects. Smoke particles of $3.4 \mu \mathrm{m}$ diameter were introduced in the airflow, giving a Stokes number $S k=5 \cdot 10^{-4}$ which indicates that the particles follow consistently the motion of the fluid. Particle images are recorded using the digital high-speed camera Phantom V1210. The laser pulsations are generated by a two cavity Nd:YLF $(527 \mathrm{~nm})$ lasers (Photonics Industries International Inc. DS-527-60) and they were redirected using mirrors to light the wake region right after the wing's trailing edge. The laser sheet with a $2.5 \mathrm{~mm}$ thickness can be seen in the mid-span section depicted in Fig. 1. The sampling rate for the acquisition was $9.5 \mathrm{KHz}$.

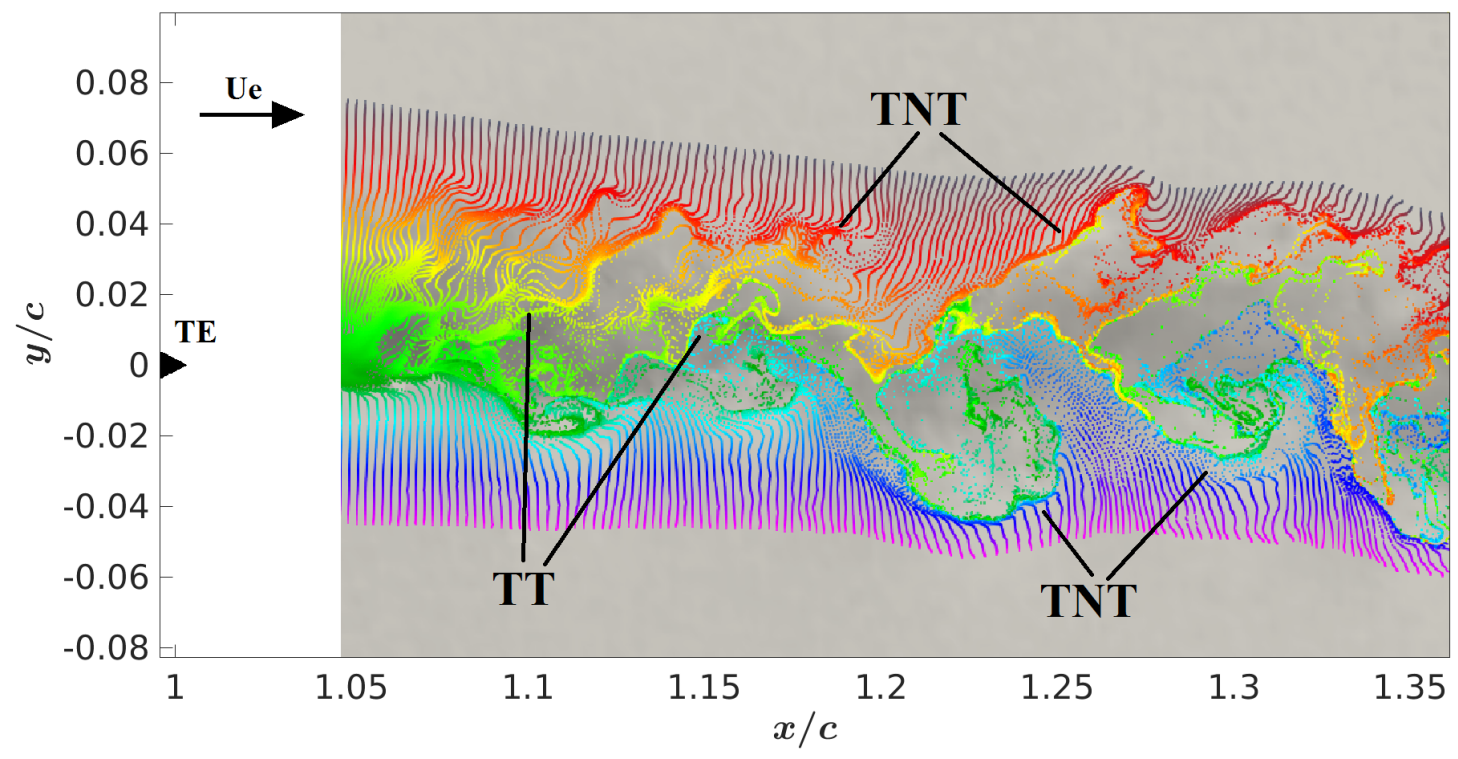

Fig. 3 Instantaneous velocity field from the TRPIV measurements, visualizations by means of streaklines for $\operatorname{Re} 1 \mathrm{M}$, angle of attack $a_{o}=10^{\circ}$; trailing edge marked with a symbol; the color of each particle represents its initial positions.

The numerical simulations have been carried out respecting the aforementioned experimental conditions. The grids were constructed depicting faithfully the geometry of test section. The stream-wise direction for the measurements is on the $x$ axis and the vertical direction is that of the $y$ axis. The $y / c=0$ value corresponds to the position of the trailing edge. For the numerical simulations, the stream-wise direction is on the $x$ axis and the vertical direction is that of the $z$ axis, leaving $y$ axis along the span-wise direction. Unlike the experimental reference system, here the $z / c=0$ value corresponds to the position of the leading edge. This is the reference system that will be used for the comparison of the computations with the experimental results.

The instantaneous velocity field measured by means of TRPIV is presented in Fig. 3. The plane presented in this figure is the one right after the wing, as presented in 1 . Numerical points were added in post-processing to follow the velocity vector measured at each time-step, providing a streakline visualization. In that sense, the color of each "particle" represents its initial positions. With the streakline visualization, the coherent and chaotic turbulent structures developed in the wake are highlighted providing a view of the dynamic behavior of the wake. The turbulent wake is restrained in between two interfacial thin shear layers, the Turbulent/Non-Turbulent (TNT) Interfaces [16, 17]. Well inside the wake region, additional Turbulent/Turbulent Interfaces develop [18]. The interfaces are indicated in Fig. 3 as captured by TRPIV measurements.

Figure 4 provides a global view over the computational domain and the solution acquired by means of numerical simulation with the M3. The flow coming from the inlet moves downstream from left to right from hereby after. Close to the leading edge, the appearance of a laminar separation bubble guides after re-attachment the transition to turbulence. The production of turbulent kinetic energy starts after $x / c=4.3 \%$ and the boundary layer at the separated trailing edge is turbulent. The quasi-steady detachment begins after $x / c \approx 80 \%$. This detachment, evidenced through streamlines, leads to a thicker effective body and an unstable wake region right after. The upper and lower shear layer instabilities interact with each other giving an irregular alternating shedding further downstream. This gives birth to secondary 
three-dimensional instabilities in the same way as studied in low Reynolds numbers around a wing [19]. The coherent vortices develop spanwisely counter-rotating cells according to a secondary instability amplification as studied in [20] and become undulated displaying predominant wavelengths that are fractions of the chord.

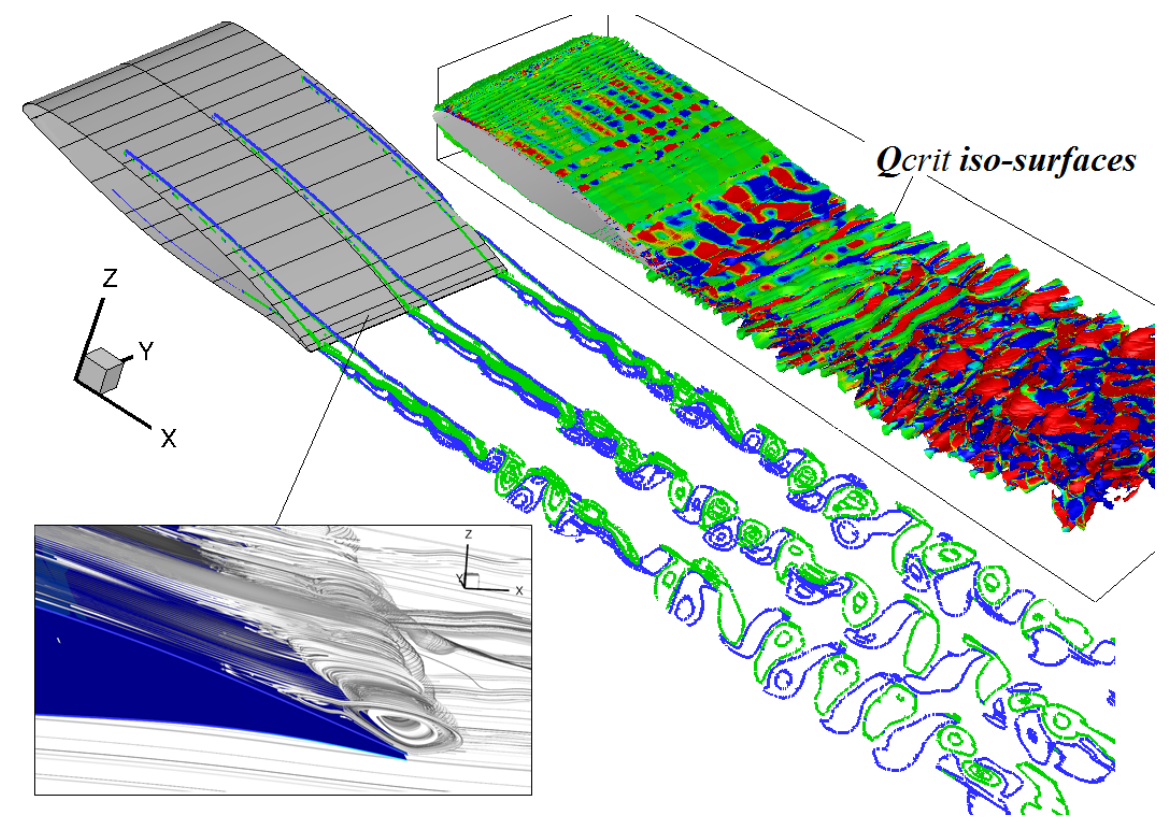

Fig. 4 Results from the 3D simulation for $\operatorname{Re} 1 \mathrm{M}$, angle of attack $a_{o}=10^{\circ}$. Birth and evolution of the primary and secondary instabilities in the wake; in the middle iso-lines of span-wise $(y)$ vorticity component; $\mathbf{Q}$ criterion iso-surfaces colored by longitudinal vorticity ranging from $-2 s^{-1}$ (blue) to $+2 s^{-1}$ (red).

The flow has a pronounced 3D character. However, the initial development of the vortical patterns in the wake has a strong apparent two-dimensional behavior. The main flow dynamics involved in the wake development can be accurately captured 2D simulation. The vortex structure dynamics and their non-linear interactions can be visualized using streaklines as computed in the wake and are pictured in Fig $5 \mathrm{a}$ Temporal signals of the vertical velocity component have been extracted in selected positions downstream of the trailing edge for both the experiments and the simulations. The corresponding Fast Fourier Transforms (FFT) of these signals have been calculated. Spectral peaks underline the main instabilities in the wake. The predominant frequency bump is representative of a coherent pattern smeared by chaotic turbulence motion, caused by non-linear interactions between the vortex shedding and the finer scale turbulent motion. This irregular formation of large scale structures is characteristic of the supercritical flow developed in this Reynolds range.

The width of the spectral bump is well captured both close to the trailing edge and further downstream. The characteristic frequency of the Kármán instability is found to be around $254 \mathrm{~Hz}$ for the experiments. The instability is a result of the interactions between the lower and upper shear layers, developed from the respective unstable boundary layers. Traces of these shear layer instabilities can also be spotted in the spectra. The lower shear layer instability is placed in a higher frequency range (around $380 \mathrm{~Hz}$ ) while the upper shear layer seems to be placed around $150 \mathrm{~Hz}$. The predominant frequencies close to the trailing edge are accurately captured by means of numerical simulation. The vortex shedding is placed around $214 \mathrm{~Hz}$, which compares well with the value obtained from the experiments. The unstable upper shear layer seems to present oscillation around $170 \mathrm{~Hz}$ while the lower shear layer is characterized by a higher frequency (above $370 \mathrm{~Hz}$ ), also in relative agreement with the PIV measurements. All the above mentioned frequencies will be referred to from hereby and after as natural frequencies as they correspond to instabilities that appear naturally in the flow.

Additionally, comparisons of the time averaged velocity profiles along the wake are depicted in Fig.6. Extracting and comparing the velocity profiles for various $x / c$ non-dimensional positions, it is evident that the agreement between the measured and computed profiles is quite satisfactory for all the grids tested. It is reminded that the reference system is the one used in the simulations. The wake expansion is accurately captured and only at the latest position the velocity deficit is slightly overestimated. After the various comparison it is proven that both the dynamic and the mean 


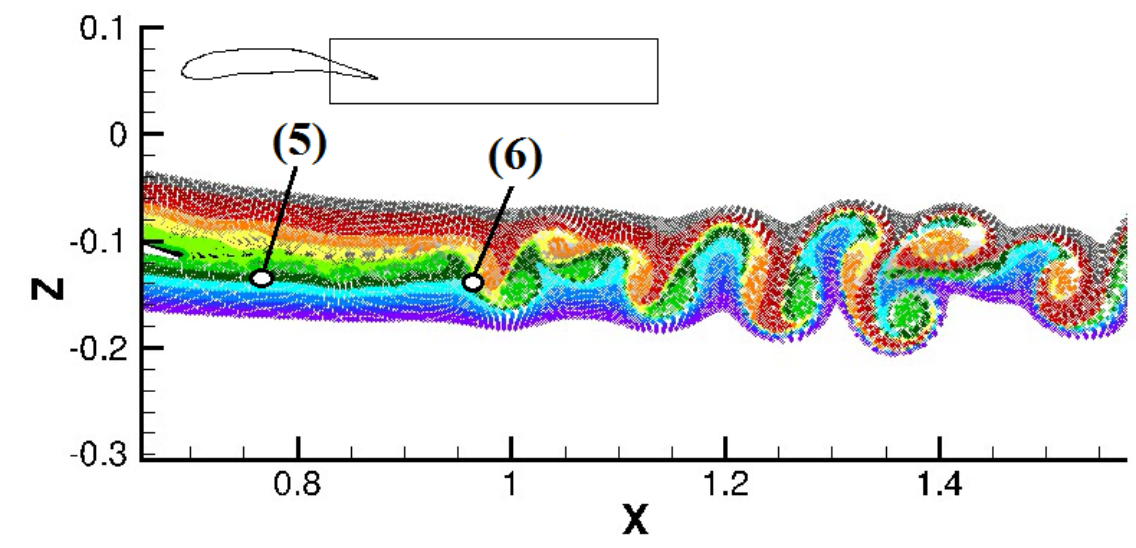

(a) Position of the monitor points in the computational domain along the wake; trailing edge tip at $\mathrm{x}=0.69$; streaklines where the color of each particle represents its initial positions.

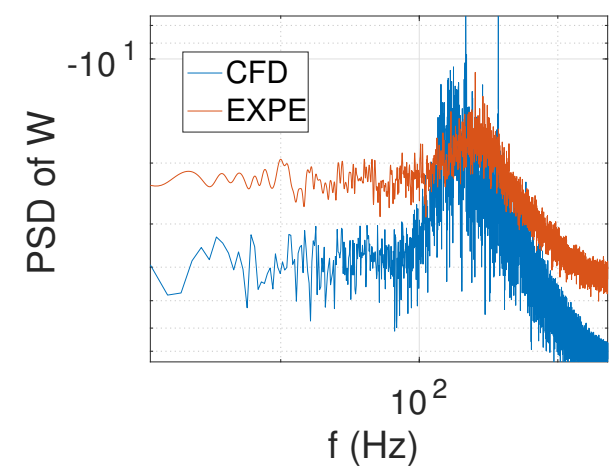

(b) Spectrum from monitor point 5 .

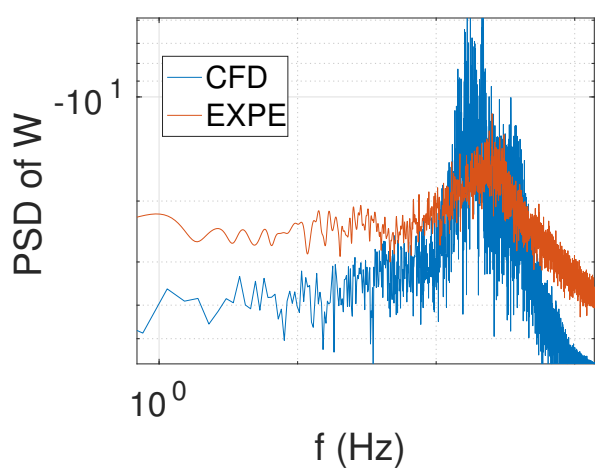

(c) Spectrum from monitor point 6 .

Fig. 5 Spectral content of vertical velocity component, comparison between computational results for the M1 grid and experiments, $\operatorname{Re} 1 \mathrm{M}$, angle of attack $a_{o}=10^{\circ}$.

characteristics of the flow are well captured even with the M1 grid, justifying, in the present study, the focus in the 2D simulations for the overall investigation and the morphing results. The two-dimensional study, allows an extensive multi-parametric study with reduced computational cost in order to assess the optimal frequency and amplitude ranges in respect of the aerodynamic performance.

\section{Sensitivity Evaluation of the Wing Geometry}

The Reynolds number is 1 million, the angle of attack of the base configuration $10^{\circ}$ and the chord length is $0.7 \mathrm{~m}$ as mentioned. The Mach number is 0.065 . The surface of the prototype is fixed for the first $71 \%$ of the chord such that the profile is only deformed where a deformable flap would be located.

The sensitivity map of the prototype is shown in Fig. 7a where positive values refer to a favorable outward deformation. In the flap region, the sensitivities indicate a clear upward motion of the upper surface except for the trailing edge where a downward deformation is predicted. On the lower surface, a strong inward i.e. upward motion of the profile is favorable except for the trailing edge where again a strong downward deformation is expected to provide an optimized shape. During the progress of the optimization, the lift increases nearly monotonically up to $18 \%$ which is shown in Fig. $7 \mathrm{~b}$ for two examples of the 60 optimization steps. However, the grid quality at the trailing edge decreases with each step, leading to heavily distorted grids for shapes giving higher lift improvements like the last step (green). This impacts the level of trust to the results for these steps. Therefore, we consider the 22nd step (red) with $10.4 \%$ lift increase as a good compromise at a reasonable grid quality. The lift increase is mainly achieved by a Gurney-flap type deformation increasing the circulation of the profile. 


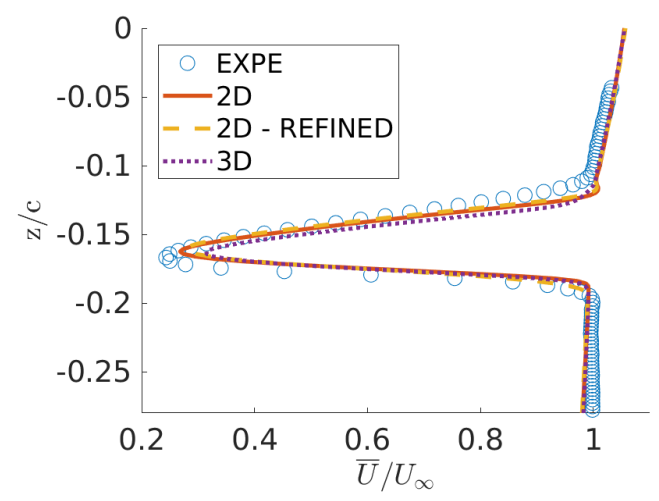

(a) $x / c=1.1$

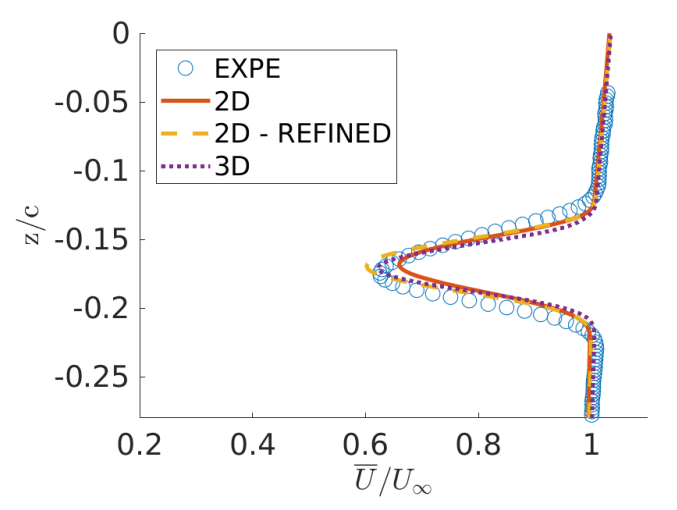

(c) $x / c=1.3$

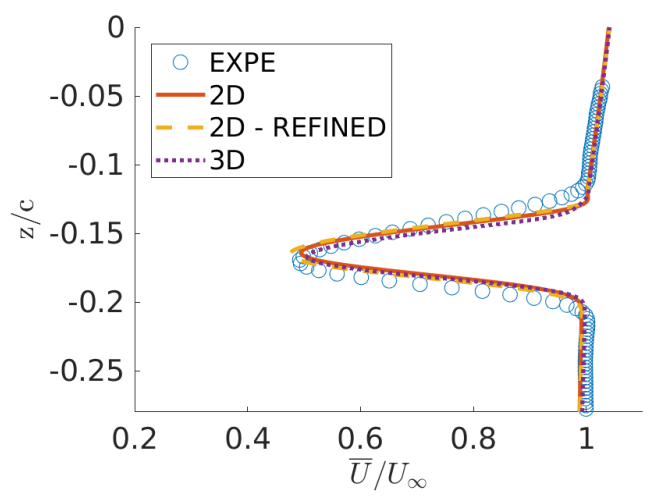

(b) $x / c=1.2$

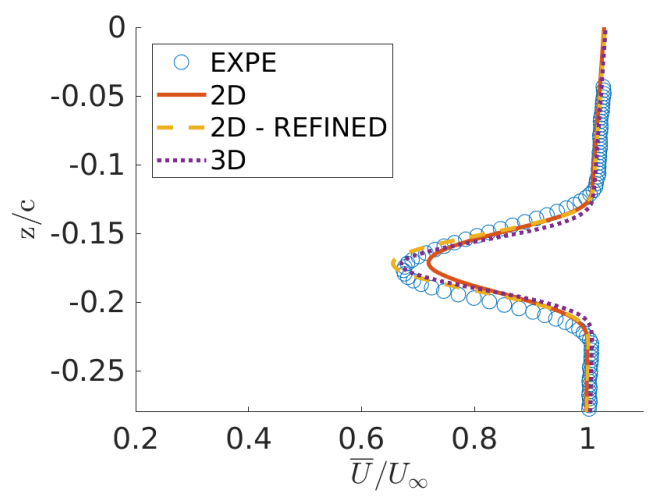

(d) $x / c=1.35$

Fig. 6 Comparison of axial time averaged velocity profiles along the wake for $\operatorname{Re} 1 \mathrm{M}$, angle of attack $a_{o}=10^{\circ}$; computational tests for the M1 mesh (2D), the refined M2 mesh (2D-REFINED) and the M3 mesh (3D) compared with the TRPIV results (EXPE).

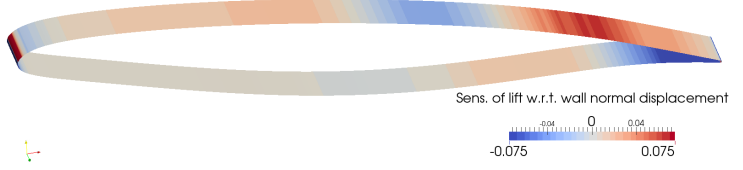

(a)

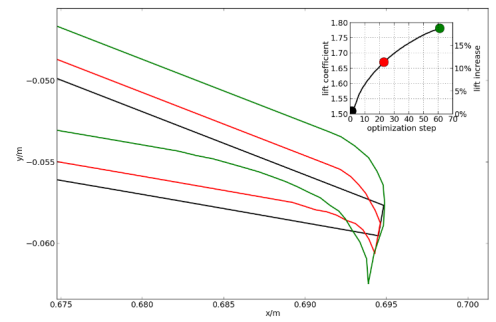

(b)

Fig. 7 Sensitivity of the lift of the lift on the full profile with respect to wall normal outward deformation (a) and optimization results close to the trailing edge (black) for the 22nd (red) and the 60th (green) optimization steps, baseline case for $\operatorname{Re} 1 \mathrm{M}$, angle of attack $a_{o}=10^{\circ}$.

\section{Numerical Investigation of Morphing}

The HFVTE system on the prototype is designed to reach amplitudes up to one millimeter. The technical characteristics for the electro-active hybrid morphing actuation are extensively described in [1,7]. The motion and slight deformation of the near-trailing edge region due to the $L_{p}=35 \mathrm{~mm}$ long MFC piezo-actuators vibrating is also applied in the numerical experiments. The Arbitrary Lagrangian-Eulerian methodology [10] is utilized for the calculation of the variables in the deformable/moving grid. The applied displacement in the trailing edge region follows exactly the second-order polynomial deformation applied on the reduced scale prototype. The frequency of the vibration $f_{a}$ and the 
amplitude $A_{o}$ of the sinusoidal time variation are left to be imposed in each test case. The time variant polynomial vertical deformation $d z$ for every surface point along the streamwise $x$ direction, is given in Eq. 1 .

$$
d z=A_{o}\left[\frac{2}{3 L_{p}^{2}}\left(x-x_{o}\right)^{2}+\frac{1}{3 L_{p}}\left(x-x_{o}\right)\right] \sin \left(2 \pi f_{a}\right)
$$

for every $x>x_{o}$, where $x_{o}=c-L_{p}$ with $c$ the chord of the airfoil and $L_{p}$ the patch length. The amplitude $A_{o}$ corresponds to the displacement of the ending tip of the trailing edge. In the tests following, the effect of the aerodynamic forces on the vibrational behavior of the piezo-actuators has not been taken into account (one-way fluid-structure interaction) since they have been evaluated as negligible in [7]. The effects using only the M1 grid will be examined as it has been proven to provide results that compare well with the experimental data.

\section{A. Frequency Variation}

The morphing has prominent effects on the development of vortical structures in the wake. The amplitude of the vibration was held constant at $0.35 \mathrm{~mm}$ while different values for the actuation frequency $f_{a}$ were tested. In this application the actuation patch length was held constant at $L_{p}=35 \mathrm{~mm}$, equal to the length of the actual piezoelectric patches used in the experiments. This amplitude value was chosen as it was the largest one to be tested during the first series of experiments with this specific morphing implementation. Visualizations by streaklines created in the same manner with the experiments, are presented in Fig. 8, comparing the effects due to the sinusoidal actuation (Figs. 8b- 8d) with the case where no morphing was applied (Fig. 8a). Respectively, in Fig. 9 contours of the ensemble-averaged $y$ vorticity component (axis vertical on the plotted plane) highlight the morphing alterations of the dynamics in the wake for various frequencies.

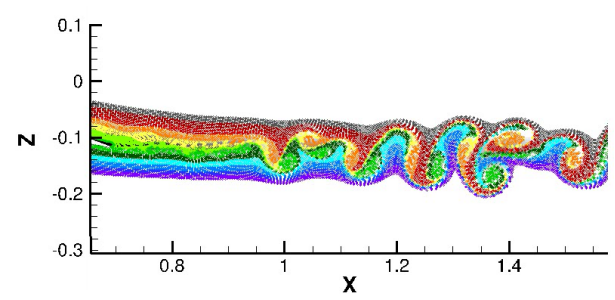

(a) No Morphing

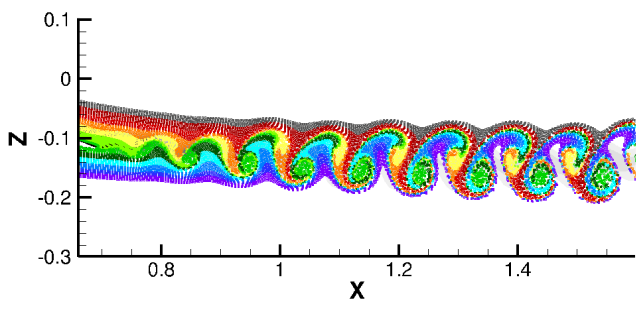

(c) $f_{a}=200 \mathrm{~Hz}$

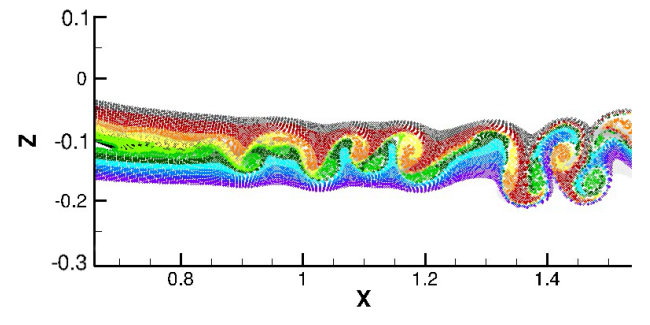

(b) $f_{a}=60 \mathrm{~Hz}$

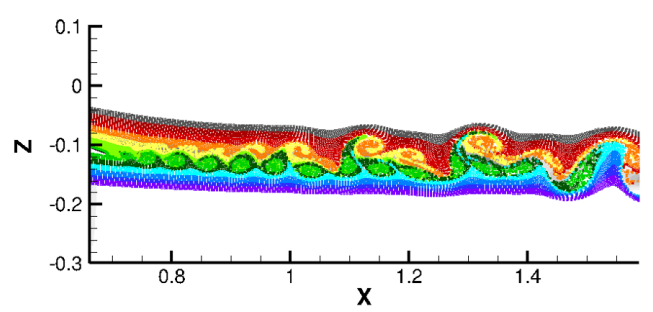

(d) $f_{a}=300 \mathrm{~Hz}$

Fig. 8 Development of vortical structures in the wake, visualization with streaklines; the color of each particle represents its initial positions. The amplitude is $0.35 \mathrm{~mm}$ for every actuating frequency.

For frequencies lower than the ones related to the shear layer instabilities and the alternating shedding (e.g. for $f_{a}=60 \mathrm{~Hz}$ in Fig. $8 \mathrm{~b}$, for $f_{a}=100 \mathrm{~Hz}$ in Fig. $9 \mathrm{~b}$, the flow coherent dynamics seem unaffected by the perturbation travelling at a much lower propagation speed. The irregular character of the flow is prevailing. Approaching the Von Kármán shedding frequency $\left(f_{a}=200 \mathrm{~Hz}\right)$, resonance phenomena take place (actually starting from $f_{a}=150 \mathrm{~Hz}$ - see Fig. 9c). The alternating vortex shedding tracks the morphing frequency, non-linear interactions are suppressed and large coherent, highly energetic structures are developed creating clearly a vortex sheet. This was first suggested in [5] where the receptivity of the flow in the wake of an airfoil was studied. It was proven that for a frequency range (namely the "locking range") around a natural frequency the measured velocity signals presented a uniform time series, 
a suppression of irregularities and an energy increase of the fluctuations. The width of this range was dependent on the amplitude of the input power of the forcing.

By further increasing the actuation frequency $\left(f_{a}=300 \mathrm{~Hz}\right)$, smaller vortices are generated in the lower shear region and get convected further downstream. These vortices of high momentum are reinforced and shielded due to the morphing and as a result they tend to remain intact ignoring the influence of the upper shear layer. The interaction with the latter is downgraded resulting to the suppression of the alternating sheading until way further downstream. The forced lower shear layer undergoes a merging process that leads to the halving of its frequency and an alternating pattern emitted at this halved frequency after $x=1.4 \mathrm{~m}$. As a result, the wake regions remains much thinner for a longer distance after the wing. Increasing the $f_{a}$ further away from the spectral bump of natural frequencies weakens the "locking mechanism" and irregularities start to reappear closer to the wing. Actuating at $f_{a}=450 \mathrm{~Hz}$ (Fig. $9 \mathrm{~g}$ ) brings back the "lock-in" phenomena probably acting as the harmonic of a natural frequency. However, the wake region remains thinner as smaller faster structures are developed. Further increasing the frequency once again leads to a change in the flow mode, the low shear layer gets mainly affected and guides the wake development.

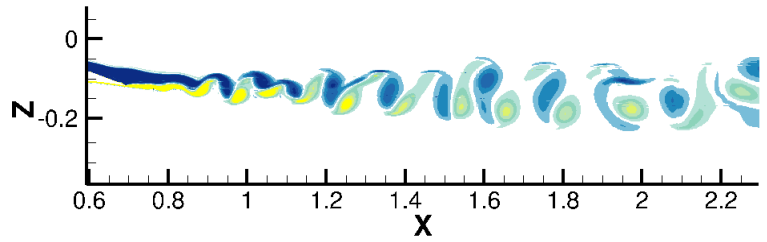

(a) No Morphing

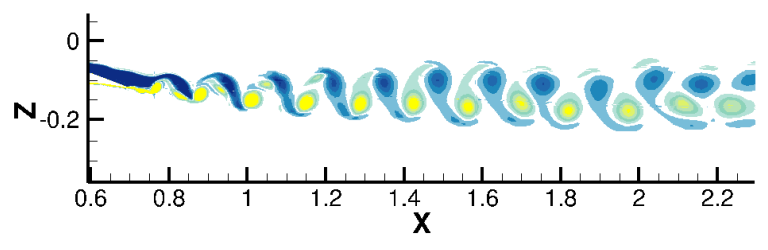

(c) $f_{a}=150 \mathrm{~Hz}$

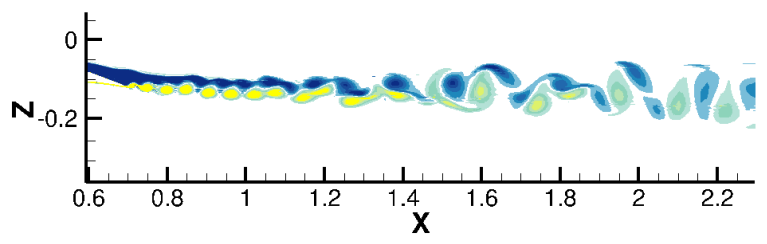

(e) $f_{a}=300 \mathrm{~Hz}$

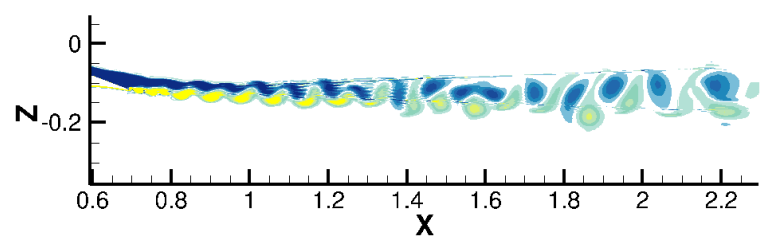

(g) $f_{a}=450 \mathrm{~Hz}$

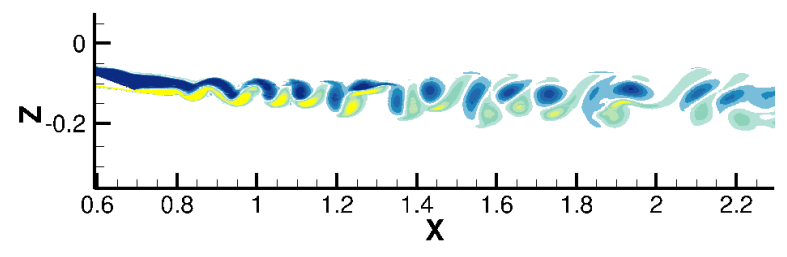

(b) $f_{a}=100 \mathrm{~Hz}$

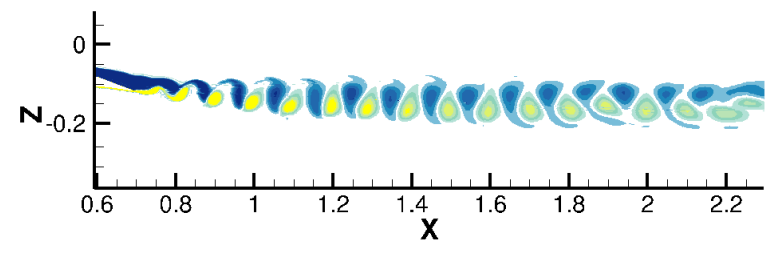

(d) $f_{a}=200 \mathrm{~Hz}$

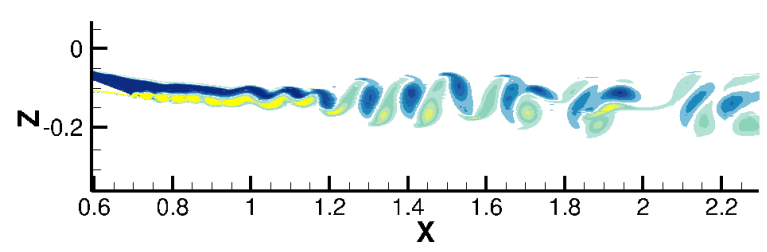

(f) $f_{a}=370 \mathrm{~Hz}$

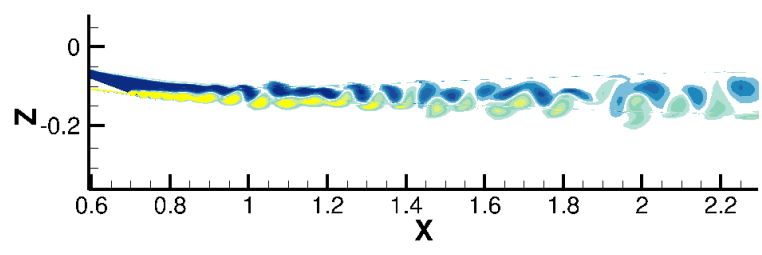

(h) $f_{a}=510 \mathrm{~Hz}$

Fig. 9 Development of vortical structures in the wake, visualization with the ensemble-averaged $y$ vorticity component. The amplitude is set at $0.35 \mathrm{~mm}$ for every actuating frequency.

An explanation of the mechanisms involved will be attempted here. Besides the upper and lower shear layers that manifest themselves as turbulent / non-turbulent (TNT) interfaces, an existing turbulent / turbulent interface (TT) is formed due to the shear caused by the detachment of the flow. This is something also noted from the PIV measurements. This interface is manipulated by the morphing applied on the trailing edge and is hypothesized that could aid to the shielding of the shear vortices created in that it provides a thin layer that de-correlates the two TNT interfaces. It has been proven in [18] that interfacial shear layers tend to act in this way. It is possible that this interface strengthens 
the low shear layer vortices with which it seems to correlate, blocks the interactions and perturbations coming from the upper shear and helps them propagate unaltered downstream. This could as well be a clear manifestation of eddy blocking phenomena taking place and preventing the generation of larger turbulent structures in the wake (as in the static case). The way this interface acts and guides the dynamics when an alternating pattern is established remains to be investigated.

In Fig. 10 the mean axial velocity profiles are plotted along the wake for various $x / c$ non-dimensional positions. Both the $z$ and the $x$ axis have a zero value at the leading edge of the wing. It is evident that for frequencies lower than the natural ones of the flow (i.e. for $60 \mathrm{~Hz}$ and $100 \mathrm{~Hz}$ ) only slight changes in the profiles are visible and mostly at the early $x / c$ stations, close to the trailing edge. For an actuation frequency close to the natural one (i.e. for $200 \mathrm{~Hz}$ ), the wake is slightly displaced at a lower height and becomes wider. For a higher frequency $(300 \mathrm{~Hz})$, a much thinner wake is visible. As the alternating shedding is suppressed until further downstream positions, the upper and lower shear layers do not spread as much, the shearing gets stronger and the velocity deficit becomes more important. For the latter morphing frequency, a smaller value of $0.15 \mathrm{~mm}$ for the amplitude was also tested. The velocity profiles undergo the exact same changes and are almost indistinguishable besides some slight differences close to the minimum values. The driving parameter is the frequency and the amplitude seems to have a secondary role as long as the same order of magnitude is maintained. The changes in the time averaged profiles attest to the displacement of the velocity deflection points. This modifies in return the most prominent modes as seen in the previous section, something that could be validated by a stability analysis.

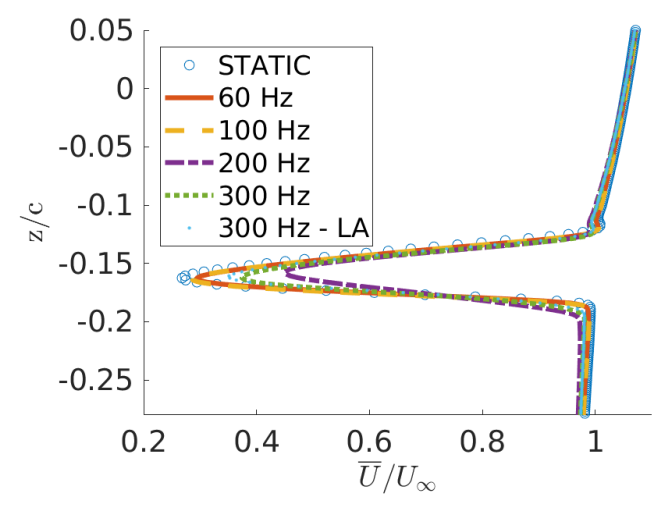

(a) $x / c=1.1$

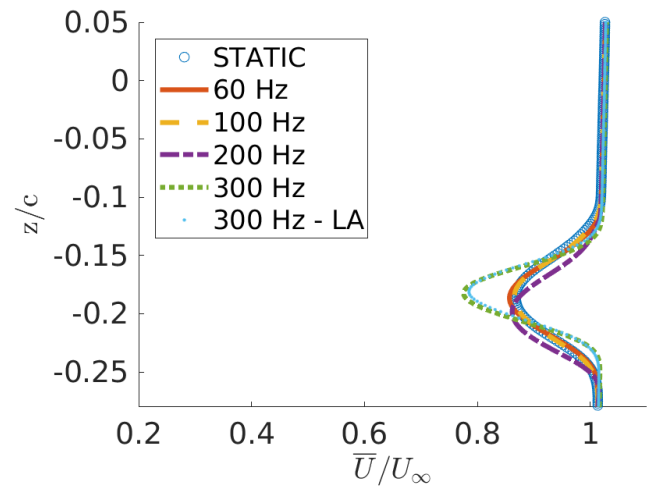

(c) $x / c=1.7$

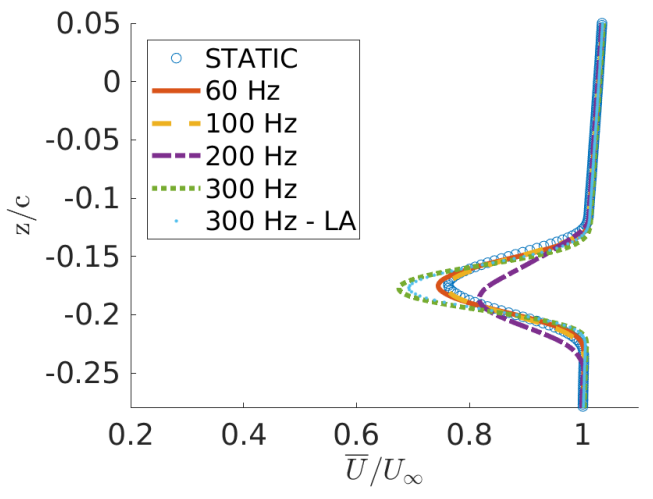

(b) $x / c=1.4$

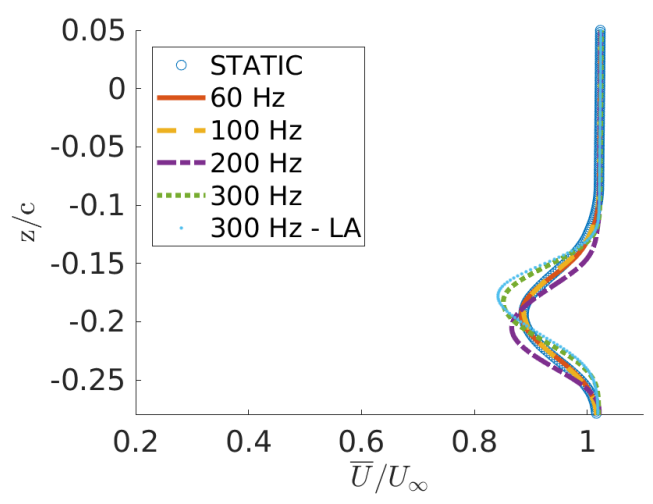

(d) $x / c=2.0$

Fig. 10 Comparison of mean longitudinal velocity profiles along the wake for various actuating frequencies; the amplitude is set at $\mathbf{0 . 3 5} \mathrm{mm}$ for every morphing application, except for the $300 \mathrm{~Hz}-\mathrm{LA}$ (lower amplitude) case where a value of $0.15 \mathrm{~mm}$ was used.

The effect on the aerodynamic forces is evaluated in Fig. 11. The effect of the actuation frequency on the lift and drag coefficients is presented for a constant amplitude of $0.35 \mathrm{~mm}$. Actuating in the region around the natural shedding frequency presents a prominent increase in both lift and drag mean values, accompanied with a significant increase in 
the fluctuations (as indicated by the root mean squared - rms - values) of the coefficients as well. This attests to the resonance previously mentioned and is also in agreement with the experimental studies included in [5]. Acting with frequencies outside this region still provides an increase in lift and in some cases a decrease in drag. The aerodynamic efficiency, expressed by the ratio of lift over $\operatorname{drag} c_{L} / c_{D}$ is always increased and at the same time the actuation keeps the rms levels in lower (acceptable) values. Approaching region of the first harmonic of the shedding frequency, the resonance phenomena reappear. Increase of the rms values can be exhibited through distinct peaks that are especially prominent in the drag evolution. In all the morphing cases the increased aerodynamic performance could not achieved with a static deformation of the trailing edge at the maximum displacements, attesting to the fact that the dynamic nature of the morphing application is responsible for the effects on the forces.

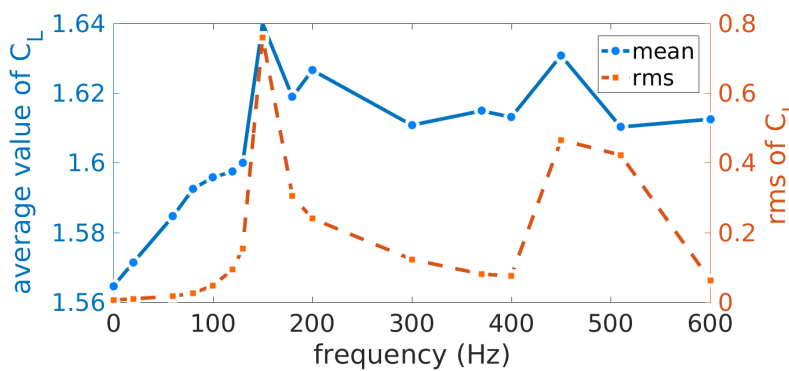

(a) Lift coefficient

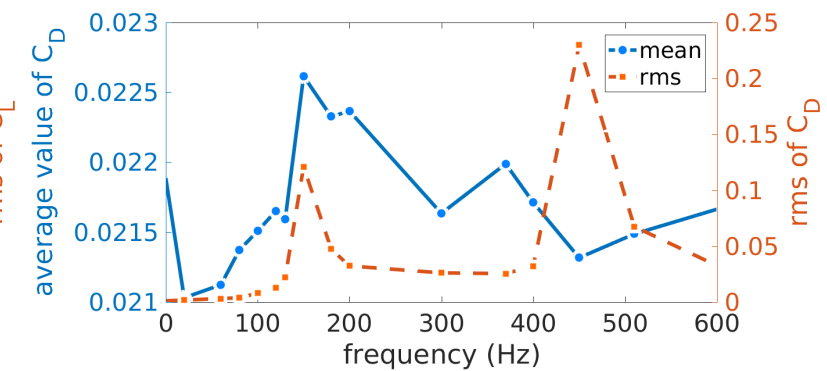

(b) Drag Coefficient

Fig. 11 Effect on the aerodynamic coefficients versus frequency variations with a constant amplitude of $0.35 \mathrm{~mm}$; zero values for frequency imply absence of morphing.

\section{B. Length Scales of Morphing}

In this section, the effect of the characteristic length scales involved in the morphing is analyzed. The amplitude $A_{o}$ and the length of the actuating patch $L_{p}$ were examined separately and in combination, for the effects they produced at specific actuation frequencies. Three frequencies in total are examined here, at values both below $(60 \mathrm{~Hz}, 100 \mathrm{~Hz})$ and above $(300 \mathrm{~Hz})$ the natural shedding frequency. The reference case P35 for which $L_{p}=35 \mathrm{~mm}$ as in the experiments was compared against cases where the length was increased. For $f_{a}=60 \mathrm{~Hz}$ and $f_{a}=100 \mathrm{~Hz}$ an $L_{p}=120 \mathrm{~mm}$ was tested (P120) while two lower values of $40 \mathrm{~mm}(\mathrm{P} 40)$ and $70 \mathrm{~mm}(\mathrm{P} 70)$ were tested for the higher frequency $f_{a}=300 \mathrm{~Hz}$. As it can be seen in Fig. 12 the effect that the patch length has on the mean velocity profiles in the wake region is minimal. Only minor changes are exhibited as the dynamics remain mainly unaffected by the change in the patch length, at least when the actuation amplitude remains the same.

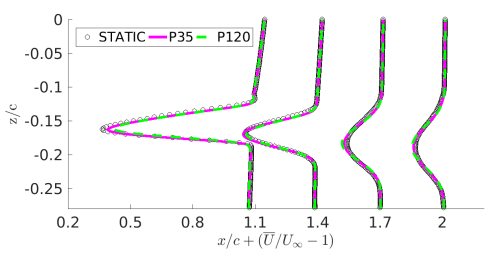

(a) $60 \mathrm{~Hz}$

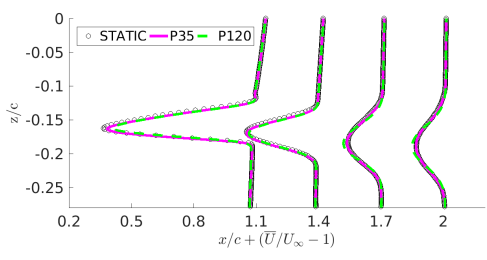

(b) $100 \mathrm{~Hz}$

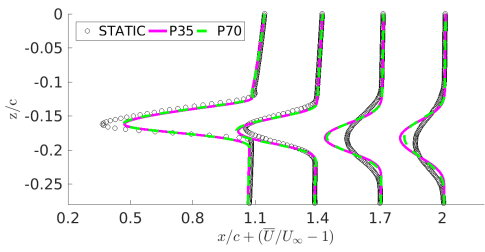

(c) $300 \mathrm{~Hz}$

Fig. 12 Comparison of mean longitudinal velocity profiles along the wake for various $L_{p}$ patch lengths; the amplitude is set at $0.35 \mathrm{~mm}$ for every morphing application.

The effect of the amplitude - in combination with the patch length - was also examined. In Figs. 13- 15 the spectral content in the wake region is examined. An FFT was performed on the vertical velocity component in the two points mentioned in Fig. 5a but also at a point at a more downstream position. Quite similar effects are examined for the lower frequencies. The combined patch - amplitude increase seems to intensify the chaotic nature of the flow which receives the amount of energy added with the actuation. The amplitude of the frequency bump is increased while it remains in the same range. The coherent picks identified also in the static case where no morphing was applied (also plotted in 
Figs. 13 and 14 for a reference) are always present in the spectrum. For the higher actuation frequency, practically no effect is exhibited in the wake dynamics due to the change of the length scales used in the morphing. The vortex dynamics and the changes examined in the previous section seems to only be dependent on the actuation frequency.

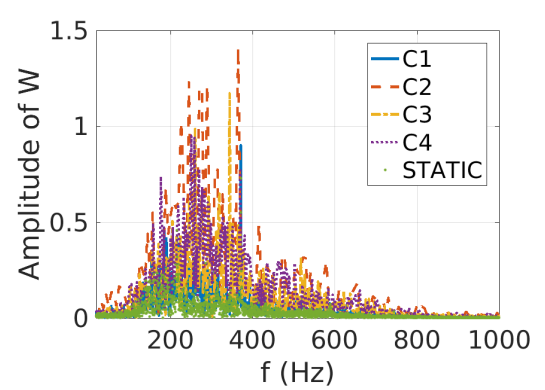

(a) Spectrum from monitor point 5 .

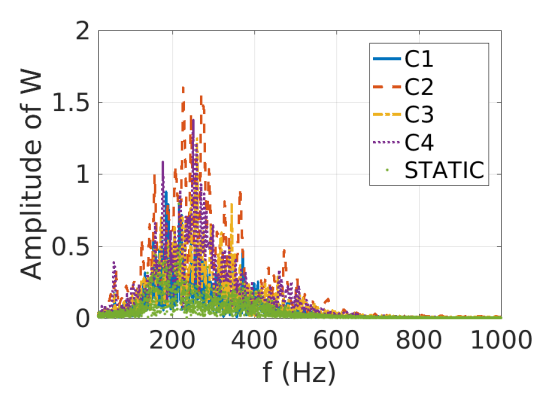

(b) Spectrum from monitor point 6.

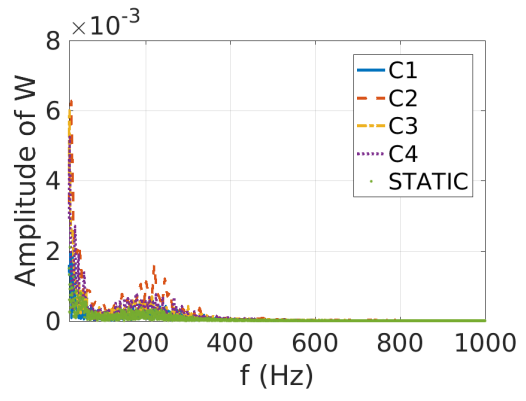

(c) Spectrum from far wake.

Fig. 13 Spectral content of vertical velocity component, actuation at $60 \mathrm{~Hz}$ for $R e 1 \mathrm{M}$, angle of attack $a_{o}=$ $10^{\circ}$ with the M1 grid; comparing P35 with $A_{o}=0.35 \mathrm{~mm}(\mathrm{C} 1), P 35$ with $A_{o}=0.7 \mathrm{~mm}(\mathrm{C} 2)$, P120 with $A_{o}=0.35 \mathrm{~mm}$ (C3) and P120 with $A_{o}=1.0 \mathrm{~mm}(\mathrm{C} 4)$. STATIC indicates no morphing applied.

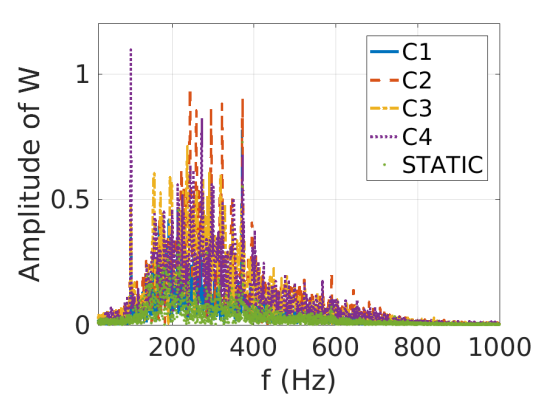

(a) Spectrum from monitor point 5 .

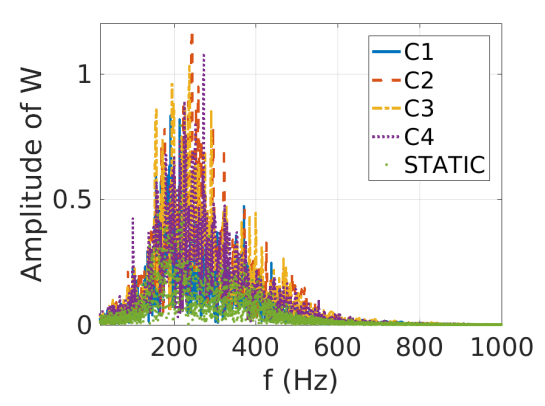

(b) Spectrum from monitor point 6.

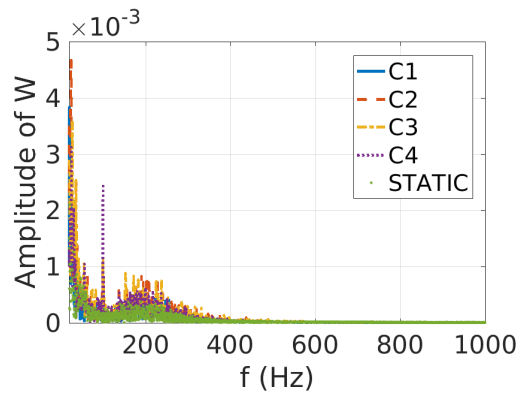

(c) Spectrum from far wake.

Fig. 14 Spectral content of vertical velocity component, actuation at $100 \mathrm{~Hz}$ for $\operatorname{Re} 1 \mathrm{M}$, angle of attack $a_{o}=$ $10^{\circ}$ with the M1 grid; comparing P35 with $A_{o}=0.35 \mathrm{~mm}(\mathrm{C1}), \mathrm{P} 120$ with $A_{o}=0.35 \mathrm{~mm}(\mathrm{C} 2)$, P120 with $A_{o}=0.5 \mathrm{~mm}$ (C3) and P120 with $A_{o}=1.0 \mathrm{~mm}(\mathrm{C4})$. STATIC indicates no morphing applied.

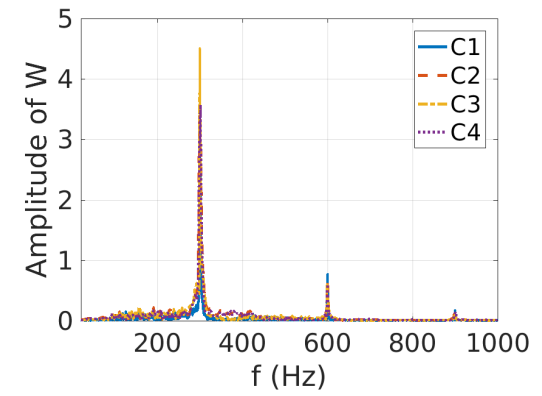

(a) Spectrum from monitor point 5 .

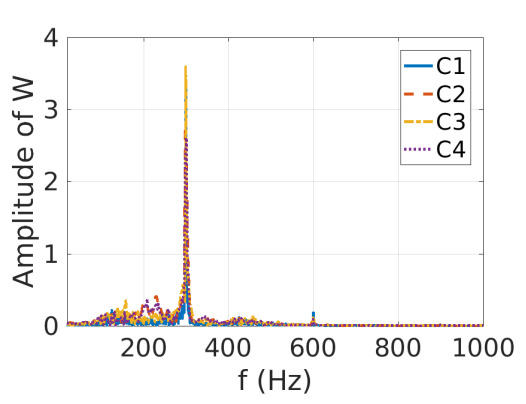

(b) Spectrum from monitor point 6.

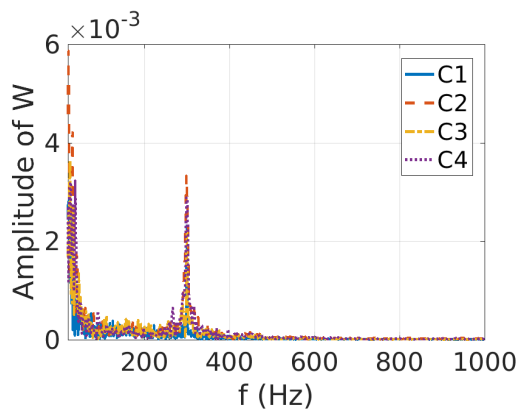

(c) Spectrum fromfar wake.

Fig. 15 Spectral content of vertical velocity component, actuation at $300 \mathrm{~Hz}$ for $\operatorname{Re} 1 \mathrm{M}$, angle of attack $a_{o}=$ $10^{\circ}$ with the M1 grid; comparing P35 with $A_{o}=0.35 \mathrm{~mm}(\mathrm{C} 1), \mathrm{P35}$ with $A_{o}=0.35 \mathrm{~mm}(\mathrm{C} 2), \mathbf{P 7 0}$ with $A_{o}=0.6 \mathrm{~mm}$ (C3) and P70 with $A_{o}=0.6 \mathrm{~mm}(\mathrm{C} 4)$.

The effect of the patch length and the amplitude variation is examined in Fig. 16 The patch length seems to provide 
only minor changes while the actuation amplitude is increased. The evolution of the forces presents a non-linear behavior with the increase of the amplitude. The lift coefficient increases up to a point and then starts decreasing again. The maximum lift value depends on the actuation frequency and its value gets a higher as the frequency increases. The drag coefficient initially decreases (at least for the lower frequencies) and then starts to increase again. After a specific amplitude, the value of the drag seems to be practically constant for the amplitudes examined in this article, it is expected hoewever to continue increasing once even higher amplitude values are examined. Whether this plateau is higher or lower than the initial drag value without morphing correlates with the frequency of the actuation. Consequently, it seems as an optimal frequency-amplitude combination exists for which a maximum lift - minimum drag ratio can be identified. No effect on the rms values is exhibited from the variation of the patch length as well. The amplitude change indicates a linear response concerning the rms values which increase with the amplitude. The slope of the rms increase depends on the actuation frequency.

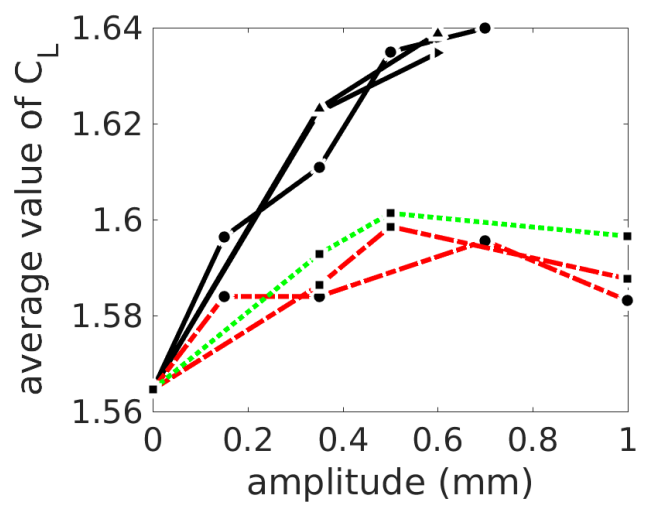

(a) Lift coefficient

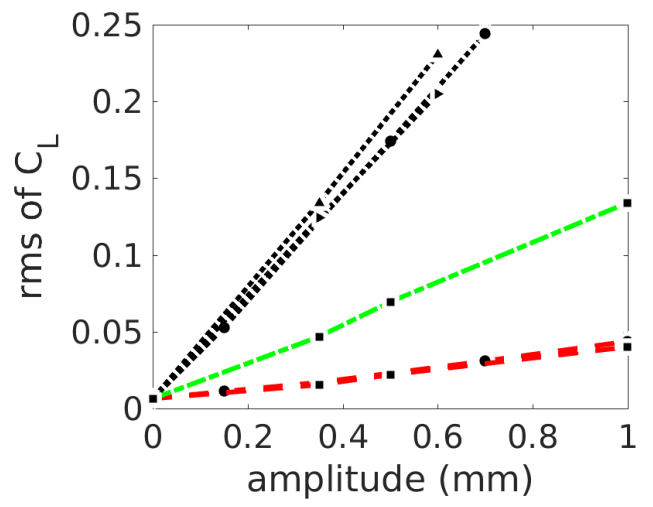

(c) Lift rms

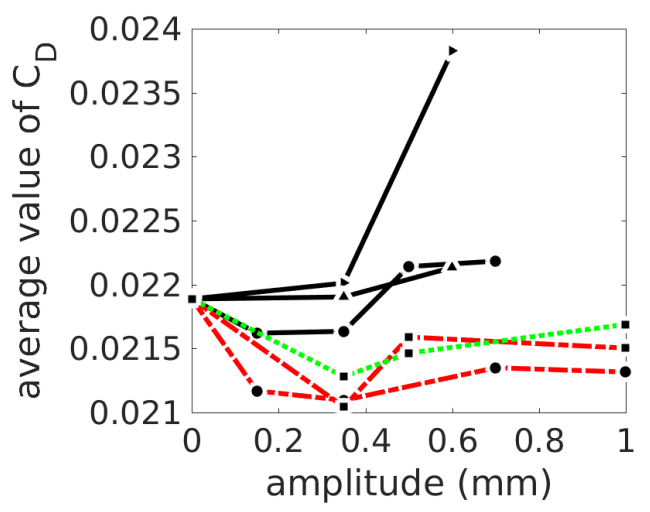

(b) Drag coefficient

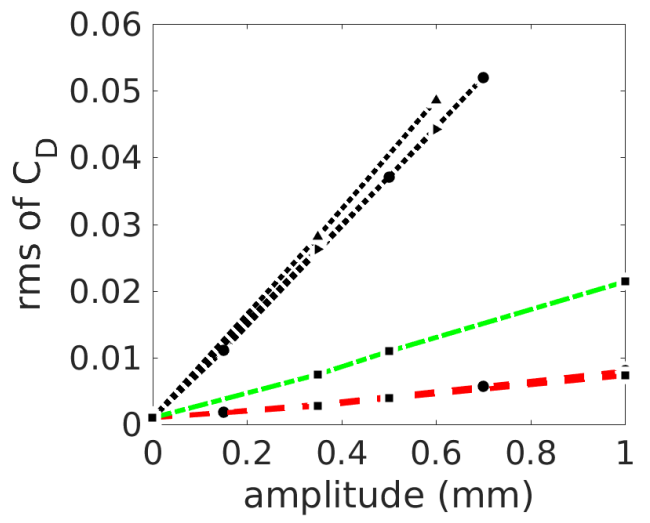

(d) Drag rms

Fig. 16 Effect on the aerodynamic coefficients versus amplitude variations for $60 \mathrm{~Hz}$ (red), $100 \mathrm{~Hz}$ (green) and $300 \mathrm{~Hz}$; zero values for frequency imply absence of morphing. With $(\bullet)$ the P35, with $(\square)$ the P120, with $(\triangle)$ the P45 and with $(\bullet)$ the P70 case.

\section{3D Morphing Effects}

In this section the three-dimensional effects of the morphing application are analyzed. Only the case with $f_{a}=300 \mathrm{~Hz}$ is examined here as it presents prominent modal changes in the wake region. The amplitude was set at the reference value of $A_{o}=0.35 \mathrm{~mm}$ once again while the length of the piezoelectric patch was $L_{p}=35 \mathrm{~mm}$. The deformation of the surface due to the electroactive morphing application was uniform along the span of the wing. A 3D view of the morphing effects on the wake can be seen in Fig. 17. The Q criterion iso-surface is plotted along the wing and in the wake region aiming at the identification of vortices. In the same figure, the vortical structures are also highlighted by means of streaklines in a mid-span slice section. 


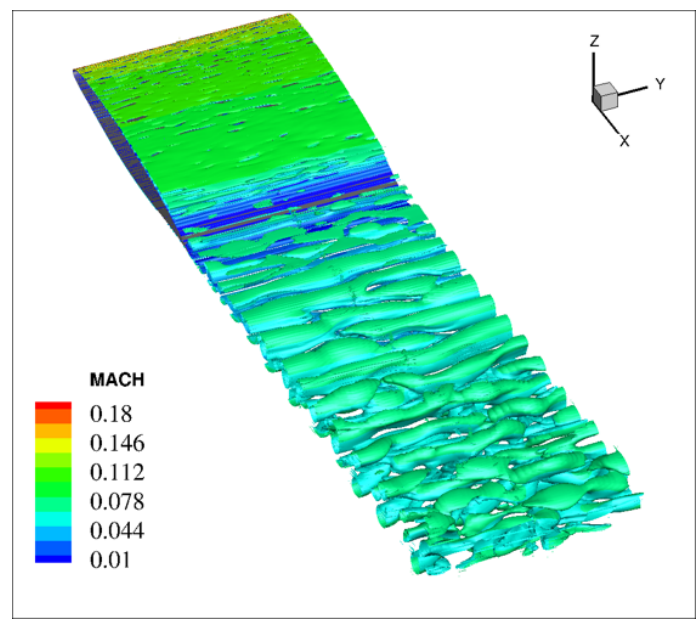

(a) Static

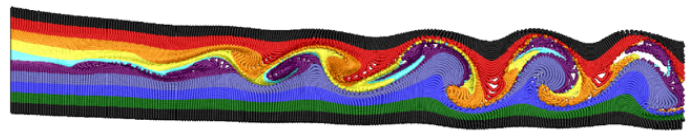

(c) Static

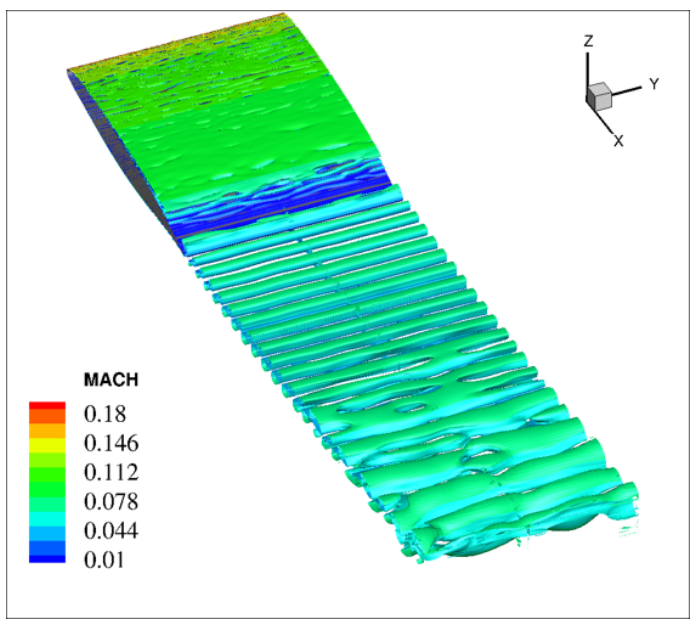

(b) $f_{a}=300 \mathrm{~Hz}$

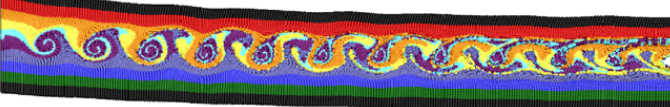

(d) $f_{a}=300 \mathrm{~Hz}$

Fig. $17 Q$ criterion iso-surface (top) for $Q=1000$ colored by the Mach value along with streakline representation (bottom) in the mid-span section; results for the non-morphing (Static) and the $f_{a}=300 \mathrm{~Hz}$ cases.

With the identification of the vortical patterns in the wake it is evidenced that the two-dimensional behavior of the flow is enhanced. Until way further downstream, the vortical tubes generated in the flow remain uniform along the span. The undulated patterns exhibited in the static (non-morphed case) cease to exist and secondary three-dimensional instabilities are suppressed. The streamwise vortex tubes - associated with the spanwise velocity fluctuations and the undulations on the iso-surface - are weakened. As a result, the chaotic wake image caused by these interactions is eliminated, at least until further downstream. The new modes emerging in the wake correlate with smaller sized vortices that - as evidenced also in the 2D simulations - keep the wake region thinner (see Figs. 17c and 17d). The weakening of the birth of three-dimensional instability modes is shown in Fig. 18. The spanwise velocity component is monitored and plotted along the span and over different vertical positions of the velocity profile at a specific streamwise $x / c$ position. With the application of the morphing, the velocity fluctuations are suppressed attesting to the two-dimensional behavior witnessed in Fig. 17 .

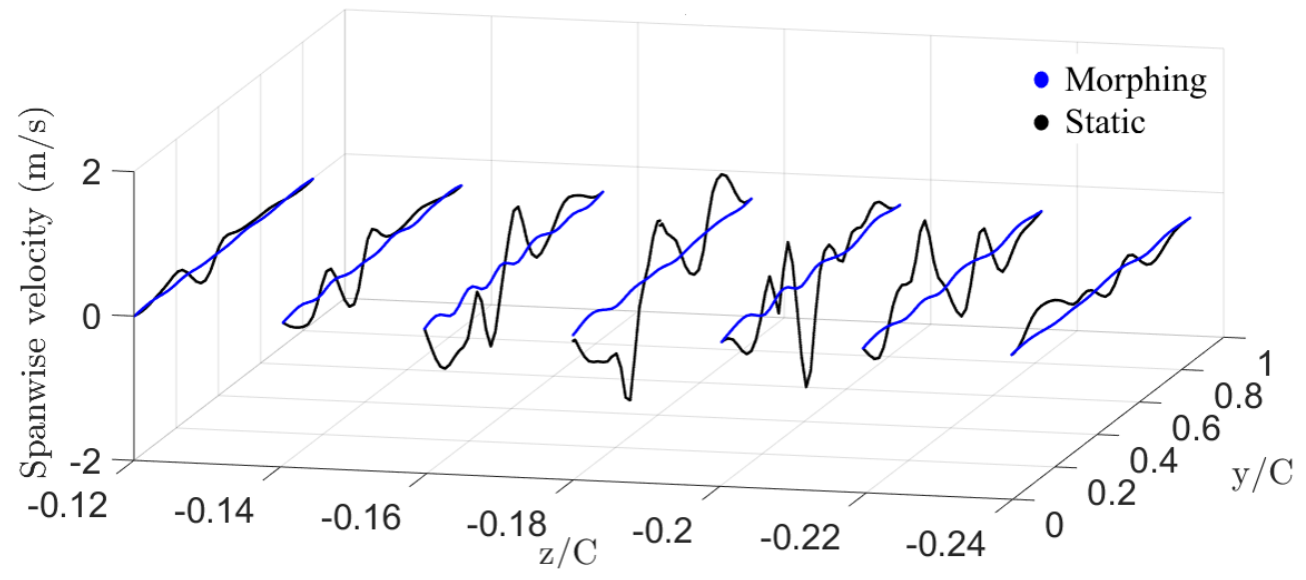

Fig. 18 Spanwise velocity component at $\mathrm{x} / \mathrm{c}=1.6$ for the non-morphing and the $f_{a}=300 \mathrm{~Hz}$ cases. 


\section{Conclusion}

In this paper the electroactive morphing effects created by Macro-Fiber Composite piezoelectric patches disposed along the span of an Airbus-A320 wing have been studied by means of TRPIV and High-Fidelity numerical simulations. These actuators introduce optimal vibrations and slight deformations of the trailing-edge region. Having available a detailed experimental database and newly acquired computational results, a combined examination of high Reynolds dynamics in the wake of this supercritical wing has been carried out in respect with the aerodynamic performance increase. The main flow characteristics have been underlined. Various frequencies and amplitude combinations have been studied numerically to evaluate the morphing effects in order to enable future experiments around the same prototype, focusing on the most optimal morphing actuations.

The wake dynamics are significantly affected by the application of morphing when acting in frequencies close or above the natural frequencies of the separated shear layers. New modes emerge and take the place of naturally existing ones. It was assessed possible to manipulate the interactions between the different instabilities and control the generation of turbulent structures in the wake. The investigation of mean wake properties has shown a thinning of the far wake region and a suppression of the alternating pattern until later downstream regions. At a given camber, the vibrating trailing edge creates small-scale turbulent eddies and adds kinetic energy in the wake which in turn causes interactions in the upper and lower shear layer. This enhances the supercritical character of the flow and producing an eddy-blocking effect constricting and strengthening (shielding) the shear layers and achieving narrower wakes [2]. By enriching this region with smaller-scale vortices, a shear sheltering effect is produced, leading to a considerable thinning of the separated shear layers, as described in [12]. The thinning of the wake region as well as feedback effects through the vorticity, enable simultaneous increase of lift and decrease of noise sources. The application of the morphing in has be proven to have the capacity to suppress three-dimensional secondary instabilities and enhance the two-dimensional behavior of the flow.

The aerodynamic performance gets enhanced as the mean value of the lift versus drag is found to be increased in every morphing case examined. By the present electroactive morphing concept, an order of $3.2 \%$ increase in lift has been achieved and at the same time a $1 \%$ decrease in the drag. The actuations at $60 \mathrm{~Hz}$ and $300 \mathrm{~Hz}$ have both achieved an increase of lift-to-drag ratio of $4 \%$. Much alike the actuation produced by the small feathers at the edges of the wings of the large-span birds, the electroactive morphing is proven capable to achieve similar effects, inspired from the nature and targeting the design of the future wings.

\section{Acknowledgments}

The authors are grateful to the Engineering and Technical services of IMFT and LAPLACE Laboratories, to the Signal and Image Services of IMFT enabling the TRPIV measurements, to the Software Services of IMFT enabling the MPI treatment of the TRPIV data bases on the supercomputer CALMIP, as well as to the three national supercomputing centers: CINES, IDRIS, CALMIP for having provided a significant CPU allocation for the present study. A special acknowledgment should be made to the SMS, "Smart morphing and Sensing for aeronautical configurations" European project (www.smartwing.org/SMS/EU, H2020 project $\mathrm{N}^{\circ}$ 723402) funded by the European Commission.

\section{References}

[1] Scheller, J., Chinaud, M., Rouchon, J., Duhayon, E., Cazin, S., Marchal, M., and Braza, M., “Trailing-edge dynamics of a morphing NACA0012 aileron at high Reynolds number by high-speed PIV," Journal of Fluids and Structures, Vol. 55, No. Supplement C, 2015, pp. 42-51. doi:10.1016/j.jfluidstructs.2014.12.012, URL http://www.sciencedirect.com/ science/article/pii/S0889974615000158

[2] Jodin, G., Motta, V., Scheller, J., Duhayon, E., Döll, C., Rouchon, J. F., and Braza, M., "Dynamics of a hybrid morphing wing with active open loop vibrating trailing edge by time-resolved PIV and force measures," Journal of Fluids and Structures, Vol. 74, No. Supplement C, 2017, pp. 263-290. doi:10.1016/j.jfluidstructs.2017.06.015, URL http://wwW.sciencedirect. com/science/article/pii/S0889974616304121.

[3] Huang, R. F., and Lin, C. L., "Vortex shedding and shear-layer instability of wing at low-Reynolds numbers," AIAA Journal, Vol. 33, No. 8, 1995, pp. 1398-1403. doi:10.2514/3.12561, URL https://doi.org/10.2514/3.12561

[4] Yarusevych, S., Sullivan, P. E., and Kawall, J. G., "On vortex shedding from an airfoil in low-Reynolds-number flows," Journal of Fluid Mechanics, Vol. 632, 2009, pp. 245-271. doi:10.1017/S0022112009007058, URL https://www . cambridge.org/core/journals/journal-of-fluid-mechanics/article/on-vortex-sheddingfrom-an-airfoil-in-low-reynolds-number-flows/FBD82B2FD88CAFB463E22F35B5D4AF55. 
[5] Gharib, M., and Williams-Stuber, K., "Experiments on the forced wake of an airfoil," Journal of Fluid Mechanics, Vol. 208, 1989, pp. 225-255. doi:10.1017/S0022112089002831, URL https://www.cambridge. org/core/journals/journal-of-fluid-mechanics/article/experiments-on-the-forced-wake-of-anairfoil/A913D3C39F7EC63E8C41AF103DD7B7DF

[6] Chinaud, M., Rouchon, J.-F., Duhayon, E., Scheller, J., Cazin, S., Marchal, M., and Braza, M., “Trailing-edge dynamics and morphing of a deformable flat plate at high Reynolds number by time-resolved PIV," Journal of Fluids and Structures, Vol. 47, 2014, pp. 41-54. URL http://dx.doi.org/10.1016/j.jfluidstructs.2014.02.007

[7] Jodin, G., Scheller, J., Rizzo, K. J., Duhayon, E., Rouchon, J.-F., and Braza, M., "Dimensionnement d'une maquette pour l'investigation du morphing électroactif hybride en soufflerie subsonique," 2015, pp. pp. 1-13. URL http://hdl.handle. net/2042/57219

[8] Hoarau, Y., Pena, D., Vos, J. B., Charbonier, D., Gehri, A., Braza, M., Deloze, T., and Laurendeau, E., "Recent Developments of the Navier Stokes Multi Block (NSMB) CFD solver," 54th AIAA Aerospace Sciences Meeting, American Institute of Aeronautics and Astronautics, ???? doi:10.2514/6.2016-2056, URL/https://arc.aiaa.org/doi/abs/10.2514/6.2016-2056

[9] Hirsch, C., Numerical Computation of Internal and External Flows: The Fundamentals of Computational Fluid Dynamics, $2^{\text {nd }}$ ed., Butterworth-Heinemann, Amsterdam, 2007.

[10] Donea, J., Giuliani, S., and Halleux, J. P., “An arbitrary lagrangian-eulerian finite element method for transient dynamic fluid-structure interactions," Computer Methods in Applied Mechanics and Engineering, Vol. 33, No. 1, 1982, pp. 689-723. doi: 10.1016/0045-7825(82)90128-1, URL http://www . sciencedirect.com/science/article/pii/0045782582901281.

[11] Bourguet, R., Braza, M., Harran, G., and El Akoury, R., "Anisotropic Organised Eddy Simulation for the prediction of non-equilibrium turbulent flows around bodies," Journal of Fluids and Structures, Vol. 24, No. 8, 2008, pp. 1240-1251. doi:10. 1016/j.jfluidstructs.2008.07.004, URL http://wwW. sciencedirect.com/science/article/pii/S0889974608000820

[12] Szubert, D., Grossi, F., Jimenez Garcia, A., Hoarau, Y., Hunt, J. C. R., and Braza, M., "Shock-vortex shear-layer interaction in the transonic flow around a supercritical airfoil at high Reynolds number in buffet conditions," Journal of Fluids and Structures, Vol. 55, 2015, pp. 276-302. doi:10.1016/j.jfluidstructs.2015.03.005, URL http://www. sciencedirect .com/science/ article/pii/S0889974615000602

[13] Braza, M., Perrin, R., and Hoarau, Y., “Turbulence properties in the cylinder wake at high Reynolds numbers,” Journal of Fluids and Structures, Vol. 22, No. 6, 2006, pp. 757-771. doi:10.1016/j.jfluidstructs.2006.04.021, URL http://wWw. sciencedirect.com/science/article/pii/S0889974606000429.

[14] Nemili, A., Özkaya, E., R. Gauger, N., Kramer, F., and Thiele, F., Discrete Adjoint Based Optimal Active Control of Separation on a Realistic High-Lift Configuration, 2016, pp. 237-246. doi:10.1007/978-3-319-27279-5_21.

[15] Hascoet, L., and Pascual, V., "The Tapenade Automatic Differentiation Tool: Principles, Model, and Specification," ACM Trans. Math. Softw., Vol. 39, No. 3, 2013, pp. 20:1-20:43. doi:10.1145/2450153.2450158, URL http://doi .acm.org/10.1145/ 2450153.2450158

[16] Hunt, J. C. R., Eames, I., Silva, C. B. d., and Westerweel, J., "Interfaces and inhomogeneous turbulence,” Philosophical Transactions of the Royal Society of London A: Mathematical, Physical and Engineering Sciences, Vol. 369, No. 1937, 2011, pp. 811-832. doi:10.1098/rsta.2010.0325, URL http://rsta.royalsocietypublishing.org/content/369/1937/811

[17] Hunt, J. C. R., Eames, I., and Westerweel, J., "Vortical Interactions with Interfacial Shear Layers," IUTAM Symposium on Computational Physics and New Perspectives in Turbulence, IUTAM Bookseries, Springer, Dordrecht, 2008, pp. 331-338. doi:10.1007/978-1-4020-6472-2_50, URL https://link.springer.com/chapter/10.1007/978-1-4020-6472-2_50

[18] Ishihara, T., Ogasawara, H., and Hunt, J. C. R., "Analysis of conditional statistics obtained near the turbulent/non-turbulent interface of turbulent boundary layers," Journal of Fluids and Structures, Vol. 53, 2015, pp. 50-57. doi:10.1016/j.jfluidstructs. 2014.10.008, URL http://www.sciencedirect.com/science/article/pii/S0889974614002230

[19] Hoarau, Y., Faghani, D., Braza, M., Perrin, R., Anne-Archard, D., and Ruiz, D., "Direct Numerical Simulation of the Three-Dimensional Transition to Turbulence in the Incompressible Flow around a Wing," Flow, Turbulence and Combustion, Vol. 71, No. 1, 2003, pp. 119-132. doi:10.1023/B:APPL.0000014931.01400.7e, URL https://doi.org/10.1023/B: APPL.0000014931.01400.7e

[20] Braza, M., Faghani, D., and Persillon, H., "Successive stages and the role of natural vortex dislocations in threedimensional wake transition," Journal of Fluid Mechanics, Vol. 439, 2001, pp. 1-41. doi:10.1017/S002211200100458X, URL https://www.cambridge.org/core/journals/journal-of-fluid-mechanics/article/successivestages-and-the-role-of-natural-vortex-dislocations-in-threedimensional-wake-transition/ B704A7018B90449A8A548327B731A88B 